\title{
HÁBITOS PARAFUNCIONAIS E SINTOMATOLOGIA DE PACIENTES SUBMETIDOS A INTERVENÇÕES BUCO-MAXILO-FACIAIS EM UMA CLÍNICA ESCOLA DE FISIOTERAPIA: UM ESTUDO RETROSPECTIVO DE 15 ANOS
}

\author{
PARAFUNCTIONAL HABITS AND SYMPTOMATOLOGY OF PATIENTS \\ SUBMITTED TO BUCO-MAXILLO-FACIAL INTERVENTIONS IN A \\ PHYSIOTHERAPY SCHOOL CLINIC: A RETROSPECTIVE STUDY OF 15 YEARS
}

\section{(iD) $\mathrm{Vi}$} iD Itana Lisane Spinato ${ }^{2}$

(iD) Micheline Freire Alencar Costa ${ }^{3}$

iD Daniela Aparecida Biasotto-Gonzalez ${ }^{4}$

iD Francisco Fleury Uchoa Santos-Júnior ${ }^{5}$

Endereço onde o projeto foi executado:

Centro Universitário Estácio do Ceará, setor de reabilitação bucomaxilofacial, unidade Moreira Campos,

Rua Vicente Linhares, nº 308 - Bairro Aldeota, Fortaleza/CE.

Número do Parecer do Comitê de Ética 2.333.85

Endereço para correspondência:

Victor Bruno Soares de Oliveira.

Rua 102, casa 418, Passaré, Fortaleza, CE, Brasil.

Telefone (085) 988113281.

vctr.fisio@gmail.com

${ }^{1}$ Centro Universitário Estácio do Ceará, Fortaleza, CE, Brasil. vctr.fisio@gmail.com

${ }^{2}$ Centro Universitário Estácio do Ceará, Fortaleza, CE, Brasil. itana.spinato@yahoo.com.br

${ }^{3}$ Centro Universitário Estácio do Ceará, Fortaleza, CE, Brasil. micheinefreire@gmail.com

${ }^{4}$ Universidade Nove de Julho, São Paulo, SP, Brasil.

dani_atm@uni9.pro.br

${ }^{5}$ Centro Universitário Estácio do Ceará, Fortaleza, CE, Brasil. drfleuryjr@gmail.com

\section{Resumo}

Objetivo: Comparar clinicamente os pacientes com desordens buco-maxilofaciais (DBMF) atendidos em uma clínica-escola de fisioterapia da cidade de Fortaleza.

Materiais e métodos: Dentre 5.357 prontuários, foram coletados os dados de 315 prontuários referentes aos pacientes acometidos por DBMF, dentre eles, 62 homens e 253 mulheres.

Resultados: $\mathrm{O}$ estudo adotou perfil predominante de pacientes do gênero feminino, solteiros, entre 21 a 30 anos, estudantes, que fizeram uso de relaxante muscular; mulheres sentiram dor irradiada, já homens, dor do tipo fina. Bruxismo/endentações/desgaste dentário foram prevalentes em ambos os sexos e os sintomas que apresentaram diferença estatística entre homens e mulheres foram cervicalgia $(\mathrm{p}=0,0051)$, cefaleia $(\mathrm{p}<0,0001)$, formigamento de membros superiores $(p=0,0371)$ e dor corporal $(p=0,0234)$.

Conclusão: Bruxismo/endentações/desgaste dentário foram os prevalentes em ambos os sexos. Entre os homens, o sintoma mais prevalente foi a dor ou cansaço ao mastigar, já entre as mulheres foi a cefaleia.

Descritores: Articulação temporomandibular. Fisioterapia. Dor

\section{Abstract}

Objective: To compare clinically patients with buccomaxillofacial disorders (DBMF) treated at a physiotherapy school clinic in the city of Fortaleza.

Materials and methods: Among 5,357 medical records, data were collected from 315 medical records for patients affected by DBMF, among them, 62 men and 253 women.

Results: The study adopted a predominant profile of female patients, single, between 21 and 30 years old, students, who used muscle relaxants; women felt irradiated pain, and men, thin pain. Bruxism/indentations/dental wear were prevalent in both sex and the symptoms that showed statistical difference between men and women were neck pain $(p=0.0051)$, headache $(p<0.0001)$, tingling of the upper limbs $(\mathrm{p}=0.0371)$ and body pain $(\mathrm{p}=0.0234)$.

Conclusion: Bruxism/indentations/dental wear were prevalent in both sex. Among men, the most prevalent symptom was pain or tiredness when chewing, while among women it was headache.

Keywords: Temporomandibular joint. Physiotherapy. Pain

\section{Cite como \\ Vancouver}

Oliveira, VBS, Spinato, IL, Costa, MFA, Biasotto-Gonzalez, DA, Santos-Júnior, FFU. Hábitos parafuncionais e sintomatologia de pacientes submetidos a intervenções buco-maxilo-faciais em uma clínica escola de fisioterapia: um estudo retrospectivo de 15 anos.

Conscientiae Saúde 2020;19(1):1-12, e18352. https://doi.org/10.5585/conssaude.v19n1.18352. 


\section{Introdução}

A articulação temporomandibular (ATM) simboliza a ligação articulada da mandíbula com a base do crânio. Esta, por sua vez, possui conexões musculares e ligamentares com a região cervical, e a união dessas estruturas forma o sistema crânio-cervico-mandibular ${ }^{(1,2)}$. Em graus de complexidade, a ATM é considerada a mais complexa do corpo humano, pois lhe é conferida a possibilidade de movimentos de abertura, oclusão, lateralidade, retrusão e protrusão e por possuir duas articulações funcionando conjuntamente conectadas à mandíbula ${ }^{(3)}$.

Um dos acometimentos da ATM é a disfunção temporomandibular (DTM), que se caracteriza por limitar a amplitude de movimento (ADM) da mandíbula e região cervical, causando déficit de força, fadiga e dor muscular, otalgia, dor orofacial, crepitação, estalido, dentre outros, as quais podem afetar crianças, adolescentes e adultos ${ }^{(4,5)}$. A somação ou o aumento desses sinais e sintomas possui como consequência a limitação do indivíduo em suas atividades fisiológicas ${ }^{(6)}$. As DTMs possuem etiologia multifatorial, inexistindo, portanto, um único fator que possa ser responsabilizado pela disfunção ${ }^{(7)}$, prevalecendo três fatores: psicocomportamentais, oclusais e neuromusculares ${ }^{(8,9)}$.

De acordo com Grazi et al. ${ }^{(10)}$, as causas mais frequentes de DTM, de origem muscular, ocorrem no intervalo entre 15 e 30 anos e, a partir de 40 anos, de origem articular; de forma geral, as maiores incidências das DTM são em indivíduos entre 20 e 45 anos. Além disso, é mais comum em mulheres do que em homens (na proporção de 5:1). Cerca de 37,5\% da população brasileira apresenta ao menos um sintoma de DTM ${ }^{(11)}$.

O aumento da atividade e da tensão dos músculos mastigatórios são induzidos por agentes psicológicos estressores, seguido de apertamento e movimentos involuntários; a continuidade do uso desses músculos provoca fadiga e mioespasmos ${ }^{(12)}$. As DTMs de causas articulares estão relacionadas a desarranjos internos (variação na posição disco-côndilo) e artralgias (condições inflamatórias locais). A característica mais comum de DTM muscular é a presença de pontos gatilhos nos músculos mastigatórios ${ }^{(13)}$.

No que se refere ao tratamento farmacológico das DTM usam-se: analgésicos não opioides (anti-inflamatórios não esteroidais - AINES), analgésicos opioides (morfina, meperidina e metadona), corticosteroides (capsaicina), ansiolíticos (diazepan), antidepressivos (amitriptilina e a clomipramina), relaxantes musculares e anticonvulsivantes (clonazepam, carbamazepina e gabapentina) ${ }^{(14)}$.

Sabendo-se do grande número de indivíduos com diagnóstico de disfunção temporomandibular e, ainda, que é uma patologia que envolve danos sociais, pessoais e 
emocionais, surgiu o interesse em realizar o estudo para investigar o perfil dos pacientes que procuraram atendimento durante 15 anos em uma clínica-escola de Fortaleza. O objetivo deste estudo foi realizar a comparação clínica dos hábitos parafuncionais e sintomatologia dos pacientes com desordens buco-maxilo-faciais atendidos na clínica escola de uma IES de Fortaleza.

\section{Materiais e métodos}

O estudo caracterizou-se como descritivo e retrospectivo. No que concerne ao meio de investigação, foi de caráter quantitativo. O presente estudo foi realizado no período entre outubro e novembro de 2017 e a coleta de dados ocorreu no mês de outubro do mesmo ano, na clínica de fisioterapia do Centro Universitário Estácio do Ceará, no setor de reabilitação bucomaxilofacial, na unidade Moreira Campos, localizada na Rua Vicente Linhares, no 308 Bairro Aldeota, Fortaleza/CE.

O estudo foi realizado mediante a aprovação do Comitê de Ética em Pesquisa do Centro Universitário Estácio do Ceará, parecer N 2.333.850. A população desta pesquisa foi composta pelos prontuários dos pacientes que já foram atendidos na clínica-escola do Centro Universitário Estácio do Ceará e pelo setor de fisioterapia bucomaxilofacial nos últimos 15 anos (2003 a 2017).

Foram triados 5.357 prontuários de pacientes sendo que, destes, participaram do estudo 315 prontuários que se encaixaram nos critérios de inclusão, ou seja, que apresentaram desordens buco-maxilo-faciais (DBMF), independentemente da idade, gênero e ocupação. Foram excluídos deste estudo os prontuários ilegíveis ou com algum dano que impossibilitassem a coleta dos dados. As variáveis abordadas neste estudo foram: idade, gênero, profissão/ocupação. Durante a coleta, houve ausência de dados de alguns prontuários quanto aos medicamentos e à queixa principal, pois não houve descrição efetiva. Os outros dados não foram analisados dessa maneira, pois não apresentaram características de dores específicas (dores inespecíficas ou não descritas). Os dados que não foram especificados também não foram computados para efeitos estatísticos.

Além disso, foram coletados dados a partir de uma ficha de avaliação que continha dados referentes a dor e tipos de medicamentos usados. Os dados foram catalogados e posteriormente submetidos a uma análise estatística descritiva (frequência absoluta e percentual). Foram utilizados ainda os testes de Fisher e Chi-Quadrado, p <0,05, por meio do 
software estatístico GraphPad Prism 8.0 para IOS, (GraphPad Software, San Diego, California USA), para serem apresentados em forma de tabelas.

\section{Resultados}

Dos 5.357 prontuários levantados, foram incluídos os dados de 315 (5,9\%) prontuários dos pacientes acometidos pelas desordens buco-maxilo-faciais (DBMF), dentre eles 62 homens $(19,7 \%)$ e 253 mulheres $(80,3 \%)$ com idades prevalentes entre 21 a 30 anos. Quanto ao estado civil, observou-se que pessoas solteiras, divorciadas e viúvas foram as mais acometidas e, quanto à ocupação, o grupo estudante, do lar e aposentado foi o que obteve maior índice, sendo $30,6 \%$ dentre os homens e $47 \%$ entre as mulheres (Tabela 1). Quanto aos hábitos parafuncionais mais prevalentes, o Bruxismo, endentações e desgaste dentário foram os hábitos parafuncionais mais referidos por ambos os gêneros (25,8\% para o masculino e 39,9\% para o feminino), seguido de estalido e crepitação: 16,1\% em homens e 34,3\% em mulheres (Tabela 2). Quanto à queixa principal, o fator pós-operatório foi predominante entre os homens (29\%), as mulheres foram mais acometidas pela dor corporal (30,4\%), sem diferenças específicas entre os gêneros (Tabela 3).

No que se refere à sintomatologia, dor ou cansaço ao mastigar e cefaleia foram os que mais se destacaram entre os indivíduos do gênero masculino $(22,5 \%)$ e do gênero feminino (45\%), respectivamente. Entretanto, as mulheres apresentam uma prevalência maior que os homens em relação a sintomas específicos, como cefaleia $(p<0,0001)$, cervicalgia $(p=0,0051)$, formigamento de membros superiores $(\mathrm{p}=0,0371)$ e dor corporal $(\mathrm{p}=0,0234)$ (Tabela 4). Quanto ao tipo de dor referida, a categoria fina/profunda/perfurante afetou 4,8\% do gênero masculino, assim como, a categoria de dor irradiada/pressão $(12,6 \%)$ foi a que mais se manifestou entre o gênero feminino. Por outro lado, homens e mulheres apresentaram percepções de dor diferentes (irradiada/pressão $\mathrm{p}=0,0002$; pontada/latejante $\mathrm{p}=0,0015$ ) (Tabela 5). Quanto ao tratamento farmacológico, ambos os gêneros fizeram mais uso de relaxantes musculares para o alívio das dores em relação aos AINES, entretanto as mulheres apresentaram maior percentual de uso de relaxantes musculares que os homens (Tabela 6). 
Tabela 1 - Caracterização sociodemográfica da população estudada referente a cada variável estudada

\begin{tabular}{|c|c|c|c|c|c|}
\hline \multirow[t]{2}{*}{ Variáveis } & \multicolumn{2}{|c|}{ Masculino $(n=62)$} & \multicolumn{2}{|c|}{ Feminino $(n=253)$} & \multirow[t]{2}{*}{ p value } \\
\hline & Sim & Não & Sim & Não & \\
\hline Gênero & $19,7 \%$ & - & $80,3 \%$ & - & - \\
\hline \multicolumn{6}{|l|}{ Idade * } \\
\hline Entre 3 a 10 anos & $5(8 \%)$ & $57(92 \%)$ & $2(0,8 \%)$ & $251(99,2 \%)$ & \multirow{6}{*}{0,0100} \\
\hline Entre 11 a 20 anos & $9(14,5 \%)$ & $53(85,5 \%)$ & $30(11,9 \%)$ & $223(88,1 \%)$ & \\
\hline Entre 21 a 30 anos & $20(32,2 \%)$ & $42(67,8 \%)$ & $82(32,4 \%)$ & $171(67,6 \%)$ & \\
\hline Entre 31 a 40 anos & $11(17,7 \%)$ & $51(82,3 \%)$ & $51(20,2 \%)$ & $202(79,8 \%)$ & \\
\hline Entre 41 a 50 anos & $8(12,9 \%)$ & $54(87,1 \%)$ & $28(11 \%)$ & $225(89 \%)$ & \\
\hline Acima de 50 anos & $8(12,9 \%)$ & $54(87,1 \%)$ & $59(23,3 \%)$ & $194(76,7 \%)$ & \\
\hline Não especificado & $1(1,6 \%)$ & $61(98,4 \%)$ & $1(0,4 \%)$ & $252(99,6 \%)$ & - \\
\hline \multicolumn{6}{|l|}{ Estado Civil / Relacionamento } \\
\hline Solteiro/Divorciado(a)/Viúvo(a) & $23(37 \%)$ & $39(62,9 \%)$ & $92(36,3 \%)$ & $161(63,6 \%)$ & \multirow{2}{*}{0,4436} \\
\hline Casado /Relacionamento Estável & $11(17,7 \%)$ & $51(82,2 \%)$ & $61(24,1 \%)$ & $192(75,8 \%)$ & \\
\hline Não especificado & $28(45,1 \%)$ & $34(54,8 \%)$ & $100(3,9 \%)$ & $153(60,4 \%)$ & - \\
\hline \multicolumn{6}{|l|}{ Ocupação } \\
\hline Estudante/Do lar/Aposentado(a) & $19(30,6 \%)$ & $43(69,3 \%)$ & $119(47 \%)$ & $134(53 \%)$ & \multirow{3}{*}{0,1050} \\
\hline Professor/CLT & $17(27,4 \%)$ & $45(72,6 \%)$ & $80(31,6 \%)$ & $173(68,4 \%)$ & \\
\hline Servidor Público & $7(11,2 \%)$ & $55(88,7 \%)$ & $15(5,9 \%)$ & $238(94 \%)$ & \\
\hline Não especificado & $19(30,6 \%)$ & $43(69,3 \%)$ & $39(15,4)$ & $214(84,5 \%)$ & - \\
\hline
\end{tabular}

*Teste Chi-quadrado ou Teste Exato de Fisher entre os resultados especificados nos prontuários. p< 0,05. Fonte: Dados dos autores.

Tabela 2 - Comparação da frequência absoluta e relativa e porcentagem dos hábitos parafuncionais e sinais clínicos distribuídos por gêneros

\begin{tabular}{lccccc}
\hline & \multicolumn{2}{c}{ Masculino $(\mathbf{n}=\mathbf{6 2})$} & \multicolumn{2}{c}{ Feminino (n=253) } & p value \\
\hline Estalido / Crepitação* & Sim & Não & Sim & Não & 0,0054 \\
Stress/Roer unha & $10(16,1 \%)$ & $52(83,8 \%)$ & $87(34,3 \%)$ & $166(65,6 \%)$ & 0,4319 \\
Masca chiclete & $7(11,3 \%)$ & $55(88,7 \%)$ & $40(15,8 \%)$ & $213(84,2 \%)$ & $>0,9999$ \\
Uso de Placa de Correção & $3(4,8 \%)$ & $59(95,1 \%)$ & $16(6,3 \%)$ & $237(93,6 \%)$ & 0,2270 \\
Ruído Não Especificado & $3(4,8 \%)$ & $59(95,1 \%)$ & $27(10,6 \%)$ & $226(89,3 \%)$ & 0,3181 \\
Ausência de Ruídos & $3(4,8 \%)$ & $59(95,1 \%)$ & $25(9,8 \%)$ & $228(90,1 \%)$ & 0,5496 \\
Edema & $5(8 \%)$ & $57(91,9 \%)$ & $14(5,5 \%)$ & $239(94,4 \%)$ & $>0,9999$ \\
Bruxismo/Endentações/Desgaste dentário* & $6(9,6 \%)$ & $56(90,3 \%)$ & $26(10,2 \%)$ & $227(89,7 \%)$ & 0,0411 \\
\hline Não especificado & $16(25,8 \%)$ & $46(74,2 \%)$ & $101(39,9 \%)$ & $152(60,1 \%)$ & - \\
\hline
\end{tabular}

*Teste Exato de Fisher entre os resultados especificados nos prontuários. $\mathrm{p}<0,05$.

Fonte: Dados dos autores. 
Tabela 3 - Comparação da frequência e porcentagem da queixa principal distribuídos por gêneros

\begin{tabular}{lccccc}
\hline & \multicolumn{2}{c}{ Masculino $(\mathbf{n}=\mathbf{6 2})$} & \multicolumn{2}{c}{ Feminino $(\mathbf{n = 2 5 3})$} & p value \\
\hline Dor Corporal & Sim & Não & Sim & Não & \\
Pós-Operatório & $14(22,5 \%)$ & $48(77,4 \%)$ & $77(30,4 \%)$ & $176(69,5 \%)$ & 0,2741 \\
Cefaleia & $18(29 \%)$ & $44(70,9 \%)$ & $58(22,9 \%)$ & $195(77 \%)$ & 0,3232 \\
Dor na ATM & $2(3,2 \%)$ & $60(96,7 \%)$ & $30(11,8 \%)$ & $223(88,1 \%)$ & 0,0577 \\
Cervicalgia & $4(6,4 \%$ & $58(93,5 \%)$ & $14(5,5 \%)$ & $239(94,4 \%)$ & 0,7623 \\
Otalgia & $5(8 \%)$ & $57(91,9 \%)$ & $9(3,5 \%)$ & $244(96,4 \%)$ & 0,1614 \\
\hline Não especificado & $2(3,2 \%)$ & $60(96,7 \%)$ & $13(5,1 \%)$ & $240(94,8 \%)$ & 0,7438 \\
\hline
\end{tabular}

*Teste Exato de Fisher entre os resultados especificados nos prontuários. p< 0,05.

Fonte: Dados dos autores.

Tabela 4 - Comparação da frequência e porcentagem dos sintomas clínicos distribuídos por gêneros

\begin{tabular}{lccccc}
\hline & \multicolumn{2}{c}{ Masculino $(\mathbf{n}=\mathbf{6 2})$} & \multicolumn{2}{c}{ Feminino $(\mathbf{n = 2 5 3})$} & p value \\
\hline Cefaleia* & Sim & Não & Sim & Não & \\
Cervicalgia* & $8(12,9 \%)$ & $54(87 \%)$ & $114(45 \%)$ & $139(54,9 \%)$ & $<0,0001$ \\
Otalgia & $13(20,9 \%)$ & $49(72 \%)$ & $101(39,9 \%)$ & $152(60 \%)$ & 0,0051 \\
Dor ou Cansaço ao Mastigar & $13(20,9 \%)$ & $49(72 \%)$ & $79(31,2 \%)$ & $174(68,7 \%)$ & 0,1215 \\
Sono Comprometido & $14(22,5 \%)$ & $48(77,4 \%)$ & $77(30,4 \%)$ & $176(69,5 \%)$ & 0,2741 \\
Formigamento de Membros & $6(9,6 \%)$ & $56(90,3 \%)$ & $20(7,9 \%)$ & $233(92 \%)$ & 0,6122 \\
Superiores* & $1(1,6 \%)$ & $61(98,3 \%)$ & $25(9,8 \%)$ & $228(90,1 \%)$ & 0,0371 \\
Dor Corporal* & & & & & 0,0234 \\
\hline Não especificado & $1(1,6 \%)$ & $61(98,3 \%)$ & $27(10,6 \%)$ & $226(89,3 \%)$ & -
\end{tabular}

*Teste Exato de Fisher entre os resultados especificados nos prontuários. $\mathrm{p}<0,05$.

Fonte: Dados dos autores.

Tabela 5 - Comparação da frequência e porcentagem dos tipos de sensação dolorosa referida pelo paciente distribuídos por gêneros

\begin{tabular}{lccccc}
\hline Variáveis & \multicolumn{2}{c}{ Masculino $(\mathbf{n}=\mathbf{6 2})$} & \multicolumn{2}{c}{ Feminino $(\mathbf{n = 2 5 3})$} & p value \\
\hline & Sim & Não & Sim & Não & \\
\hline Irradiada/Pressão* & $2(3,2 \%)$ & $60(96,7 \%)$ & $57(22,5 \%)$ & $196(77,5 \%)$ & 0,0002 \\
Pontada/Latejante* & $2(3,2 \%)$ & $60(96,7 \%)$ & $48(19 \%)$ & $205(81 \%)$ & 0,0015 \\
Fina/Profunda/Perfurante & $3(4,8 \%)$ & $59(95,1 \%)$ & $23(9 \%)$ & $230(90,9 \%)$ & 0,4383 \\
\hline Não especificado & $55(88,7 \%)$ & $7(11,2 \%)$ & $147(58,1 \%)$ & $106(41,9 \%)$ & -
\end{tabular}

*Teste Exato de Fisher entre os resultados especificados nos prontuários. $\mathrm{p}<0,05$.

Fonte: Dados dos autores. 
Tabela 6 - Comparação da frequência e porcentagem do uso de medicamentos dos pacientes com Disfunção Temporomandibular distribuídos por gêneros

\begin{tabular}{lccccc}
\hline Variáveis & \multicolumn{2}{c}{ Masculino $(\mathbf{n}=\mathbf{6 2})$} & \multicolumn{2}{c}{ Feminino $(\mathbf{n = 2 5 3})$} & p value \\
\hline Relaxante Muscular* & Sim & Não & Sim & Não & \\
AINES & $5(8 \%)$ & $57(91,9 \%)$ & $68(26,8 \%)$ & $185(73,1 \%)$ & 0,0012 \\
\hline Não especificado & $2(3,2 \%)$ & $60(96,7 \%)$ & $20(7,6 \%)$ & $233(92 \%)$ & 0,2700 \\
\hline
\end{tabular}

*Teste Exato de Fisher entre os resultados especificados nos prontuários. $\mathrm{p}<0,05$.

Fonte: Dados dos autores.

\section{Discussão}

Este estudo encontrou que as DBMF foram mais presentes em mulheres, entre 21 a 30 anos de idade, solteiras e estudantes. Os hábitos parafuncionais e achados mais prevalentes foram o bruxismo/endentações/desgaste dentário. A queixa principal que os levou a procurar o serviço de fisioterapia mais prevalente, entre os homens, foi o pós-operatório; em contrapartida, as mulheres relataram mais a dor corporal. A tipologia de dor mais referida pelos homens que souberam descrevê-la foi a fina/profunda/perfurante, já para as mulheres, as dores irradiadas/pressão fizeram-se mais presentes. O relaxante muscular foi o fármaco mais utilizado por ambos os gêneros perante o tratamento farmacológico.

Os dados da presente pesquisa estão de acordo com os resultados de Karibe et al. ${ }^{(15)}$ : dos 179 pacientes, 84,4\% eram do gênero feminino e a variação de idade foi entre os 10 e 84 anos, esses resultados também concordam com os resultados de Moura et al. ${ }^{(16)}$ e Matta e Honorato $^{(17),}$ que observaram incidência de DTM acima de $80 \%$ em mulheres entre 21 e 29 anos. Além disso, Ryan et al. ${ }^{(18)}$ e Grossi et al. ${ }^{(19)}$ sugerem que as pessoas do gênero feminino são sujeitas a maior prevalência e intensidade da DTM devido a acometimentos psicossociais, quando comparadas às pessoas do sexo masculino.

Ainda com base nos dados do estudo de revisão de Ryan et al. ${ }^{(18)}$, o pico da prevalência da variação da idade de pessoas com DTM foi entre os 25 e os 45 anos de idade. Em contrapartida, os dados obtidos por Ribeiro et al. ${ }^{(20)}$ mostram que $42 \%$ das pessoas acometidas pela DTM estavam entre 46 e 60 anos de idade, discordando dos dados obtidos no presente estudo.

Além disso, Moura et al. ${ }^{(16)}$ indicam que o estado civil e a ocupação das pacientes mais acometidas são solteira e estudante, respectivamente, indo ao encontro dos resultados do presente estudo. Assim como também os resultados encontrados por Jahandideh et al. ${ }^{(21)}$, que mostram que cerca de $90,8 \%$ dos pacientes com DTM apresentaram-se como solteiros. Por outro lado, Reiter et al. ${ }^{(22)}$ descrevem que cerca de $42,3 \%$ dos pacientes eram casados, 
divergindo dos dados encontrados no presente estudo. Quanto à característica da dor mais influente, houve um destaque para a forma pulsátil em estudo prévio ${ }^{(23)}$, contrastando com os resultados do nosso estudo. Em outro estudo foram constatados, em 100\% dos pacientes com DTM associada a artralgia, dores em queimação ${ }^{(24)}$ ou ritmadas, em períodos ou intermitentes, sugerindo, desta forma, intervalo entre os episódios dolorosos ${ }^{(26)}$.

Independentemente do mecanismo e direção da associação entre as parafunções e a dor, as parafunções podem favorecer a presença da cefaleia e vice-versa ${ }^{(27)}$, esse fato corrobora com nossos achados, uma vez que a cefaleia foi um sintoma presente dentre os avaliados, e mais frequente entre as mulheres. Já com relação aos pacientes que tinham DTM e cervicalgia crônica, estes apresentam mais áreas de dor muscular e hiperalgesia ${ }^{(28,29)}$. Além disso, a presença local e difusa da dor corporal em adolescentes com DTM foram mais prevalentes quando comparados aos adolescentes que não possuíam DTM ${ }^{(30)}$. Em nosso estudo, esse dado foi descrito, de modo mais abrangente, como sendo um elemento de dor corporal, e novamente mais frequente entre mulheres. Com relação aos hábitos parafuncionais, a literatura apresenta que o gênero não impacta na prevalência do bruxismo, hábitos parafuncionais ou DTM ${ }^{(29)}$, o que difere de nossa amostra, pois a mesma apresentou mais hábitos de bruxismo entre as mulheres.

Com relação ao uso de medicamentos, a combinação entre o uso de AINEs e relaxantes musculares foi o tratamento farmacológico predominante entre os pacientes acometidos pela DTM, seguidos pelo uso somente dos AINEs e, em terceiro lugar, o uso de apenas relaxantes musculares ${ }^{(25)}$. De acordo com os apontamentos de Ouanounou et al. ${ }^{(31)}$, a melhor evidência clínica para o uso de fármacos para o tratamento da DTM é o uso dos AINEs, muito embora existam altos riscos em seu consumo, em algumas populações. Em relação ao presente estudo, os relaxantes musculares obtiveram predominância entre as opções de fármacos para alívio da dor da DTM, ou seja, os fármacos miorrelaxantes tendem a ser os mais escolhidos para o tratamento dessa disfunção. Esse foi um resultado inesperado em nosso estudo, haja vista que a seletividade do uso de fármacos entre um determinado gênero foi evidenciada e deve ser alvo de investigação mais ampla em novos estudos sobre o tema.

\section{Limitações do estudo, pontos fortes e perspectivas futuras}

O que mais dificultou a realização deste trabalho foi a não especificação de alguns dados nos prontuários da clínica escola. Por outro lado, o tempo de coleta dos dados foi determinante para uma visão geral dos achados clínicos e pode ser entendido como um ponto forte, pois trata- 
se de uma ampla perspectiva da condição patológica ao longo do tempo, 15 anos. Portanto, sugere-se que a abordagem dos avaliadores seja a mais empática possível, a fim de transmitir segurança aos pacientes e, portanto, criar uma boa aliança terapêutica. Desse modo, acreditamos que os avaliados possam relatar seus dados com menos receio para os avaliadores estudantes, assim como um maior treinamento avaliativo para a coleta de dados, envolvendo questões como entrevistas motivacionais e avaliações sequenciadas.

\section{Conclusão}

Os pacientes com desordens buco-maxilo-faciais atendidos em uma clínica-escola de fisioterapia da cidade de Fortaleza, adotaram o perfil predominante do gênero feminino, solteiras, com faixa etária entre 21 e 30 anos e estudantes. Além disso, os hábitos parafuncionais, tais como a crepitação/estalido, bruxismo/endentações e desgaste dentário foram os prevalentes em ambos os gêneros. Já quanto aos sintomas, os que apresentaram diferença entre gêneros foram cervicalgia, cefaleia, formigamento de membros superiores e dor corporal. Entre os homens o sintoma mais prevalente foi a dor ou cansaço ao mastigar; já entre as mulheres, a cefaleia.

\section{Contribuição dos autores}

Cada autor contribuiu individual e significativamente para o desenvolvimento deste artigo. Victor Bruno Soares de Oliveira (0000-0001-9307-3441): revisão do estudo, pesquisa bibliográfica, revisão bibliográfica, coleta de dados, análise de dados, escrita e formatação. Itana Lisane Spinato (0000-0002-3010-640X): revisão do estudo, revisão bibliográfica, análise de dados e formatação. Micheline Freire Alencar Costa (0000-0002-0307-1790): revisão do estudo, revisão bibliográfica, pesquisa bibliográfica, análise de dados e formatação. Daniela Aparecida Biasotto-Gonzalez (0000-0002-8498-0557): revisão do estudo, revisão bibliográfica, análise de dados e formatação. Francisco Fleury Uchoa Santos-Júnior (00000002-5685-4297): revisão do estudo, revisão bibliográfica, análise de dados, escrita, formatação e aprovação final do estudo. 


\section{Referências}

1. Okeson JP. Etiologia dos Distúrbios Funcionais do Sistema Mastigatório. In: Fundamentos de Oclusão e Desordens Temporo-Mandibulares. 2.ed. São Paulo: Arte Médicas; 1992.

2. Amantéa DV, Novaes AP, Campolongo GD, Barros TP. A importância da avaliação postural no paciente com disfunção da articulação temporomandibular. Acta Ortop Bras. 2004; 12(3): 155-59.

3. Donnarumma MDC, Muzilli CA, Ferreira C, NEMR K. Disfunções temporomandibulares: sinais, sintomas e abordagem multidisciplinar. Rev. CEFAC. 2010; 12(5): 788-94.

4. Okeson JP. Tratamento das desordens temporomandibulares e oclusão. 4.ed. São Paulo: Artes Médicas; 2000.

5. Oliveira SLS, Carvalho DS. Cefaleia e articulação temporomandibular (ATM). Rev. Neurociências. 2002; 10(3): 141-52.

6. Menezes MS, Bussadori SK, Fernandes KPS, Biasotto-Gozales DA. Correlação entre cefaleia e disfunção temporomandibular. Fisioterapia e Pesquisa. 2008;15(2):183-87.

7. Steenks MH, Wijer A. Disfunções da articulação temporomandibular do ponto de vista da fisioterapia e da odontologia - diagnóstico e tratamento. Editora Santos, 2005.

8. Melo GM, Barbosa FS. Parafunção x DTM: a influência dos hábitos parafuncionais na etiologia das desordens temporomandibulares. POS- Prespect. Oral Sci. 2009; 1(1): 43-48.

9. Carrara SV, Conti PCR, Barbosa JS. Termo do $1^{\circ}$ Consenso em Disfunção Temporomandibular e dor Orofacial. Dental Press J Orthod. 2010; 15(3):114-20.

10. Grazi RCD, Bankoff ADP, Zamai CA. Alterações posturais relacionadas com a disfunção da articulação temporomandibular e seu tratamento. Movimento \& Percepção. 2006;6(8): 15062.

11. Gonçalves DA, Speciali JG, Jales LC, Camparis CM, Bigal ME. Temporomandibular symptoms, migraine and chronic daily headaches in the population. Neurology. 2009; 73(8): 645-46.

12. Lupoli T.A, Lockey RF. Temporomandibular dysfunction: an often overlooked cause of chronic headaches. Ann Allergy Asthma Immunol. 2007; 99(4): 314-18.

13. Franco AL. Estudo da prevalência de cefaleias primárias e da sua associação com a dor orofacial em pacientes com disfunção temporomandibular crônica [dissertação]. Araraquara: UNESP: Universidade Estadual Paulista; 2009.

14. Melo GM. Terapia farmacológica em disfunções temporomandibulares: uma breve revisão. Revista Dentísticaonline. 2011; 10(21): 35-40.

15. Karibe H, Goddard G, Shimazu K, Kato Y, Warita-Naoil S, Kawakami T. Comparison of self-reported pain intensity, sleeping difficulty, and treatment outcomes of patients with 
myofascial temporomandibular disorders by age group: a prospective outcome study. BMC Musculoskeletal Disorders. 2014; 15(423): 2 - 6.

16. Moura WP, Silva PLP, Lemos GA, Bonan PRF, Montenegro RV, Batista AUD. Revisão retrospectiva de pacientes encaminhados a um serviço de disfunção temporomandibular de uma universidade pública brasileira. Rev. Dor. 2017; 18(2): 128-34.

17. Matta MAP, Honorato DC. Uma abordagem fisioterapêutica nas desordens temporomandibulares: estudo retrospectivo. Rev. Fisioter. Univ. 2003; 10(2): 77-83.

18. Ryan J, Ahkter R, Hassan N, Hilton G, Wickham J, Ibaragi S. Epidemiology of Temporomandibular Disorder in the General Population: a Systematic Review. Adv. Dent \& Oral Health. 2019; 10(3): 1-13.

19. Grossi PK, Bueno CH, Silva MAA, Pellizer EP, Grossi ML. Epidemiology of Temporomandibular Disorder in the General Population: a Systematic Review. International Journal of Prosthodontics. 2018; 31(6): 543-551.

20. Ribeiro MC, Meusel LDZ, Gaviolli E; Silveira AM, Cericato GO. Prevalence of TMJ Pain Symptom in Adults and its Association with Predisposing Factors. Biosci. J. 2018; 34(6): 18151823.

21. Jahandideh Y, Basirat M, Kananian B. Risk Factors for Temporomandibular Joint Disorder in Dental Students. J Dentomaxilofacial Radiol Pathol Surg. 2018; 7(1):23-28.

22. Reiter S, Emodi-Perlman A, Goldsmith C. Comorbidity Between Depression and Anxiety in Patients with Temporomandibular Disorders According to the Research Diagnostic Criteria for Temporomandibular Disorders. Journal of Oral \& Facial Pain and Headache. 2015; 29(2): $135-43$.

23. Dantas AMX, Santos EJL, Vilela RM, Lucena LBS. Perfil epidemiológico de pacientes atendidos em um Serviço de Controle da Dor Orofacial. Rev Odontol UNESP. 2015 Nov-Dec; 44(6): 313-319.

24. Molina OF, Aquilino RN, César EW, Cury SE, Marçal RL, Miranda MM. Tmj Arthralgia: a rarely described internal joint derangement and characterization regarding factors of age, pain description, and prevalence in craniomandibular disorders individuals (Cmds). Cadernos UniFOA. 2010; 14(5): 71-81.

25. Portinho CP, Collares MVM, Faller GJ, Fraga MM, Pinto RA. Perfil dos pacientes com disfunção temporomandibular. Arquivos Catarinenses de Medicina. 2012; 41(Sup 1): 95 - 99.

26. Pedroni CR, Oliveira AS, Bérzin F. Pain characteristics of temporomandibular disorder: a pilot study in patients with cervical spine dysfunction. J Appl Oral Sci. 2006 Out;14(5):388-92.

27. Di Paolo C, D’Urso A, Papi P, Di Sabato F, Rosella D, Pompa G, Polimeni A. Temporomandibular Disorders and Headache: A Retrospective Analysis of 1198 Patients. Pain Reasearch and Management. 2017; 2017(2): 1-8. 
28. Muñoz-García D, López-de-Uralde-Villanueva I, Beltran-Alacreu H, Fernández-Carnero J. Patients with Concomitant Chronic Neck Pain and Myofascial Pain in Masticatory Muscles Have More Widespread Pain and Distal Hyperalgesia than Patients with Only Chronic Neck Pain. Pain Medicine. 2017; 18(3): 526-537.

29. Rubin PF, DMD AE, DMD BP, DMD RBW, DMD EW. Prevalence of Bruxism and Temporomandibular Disorders Among Orphans in Southeast Uganda: A Gender and Age Comparison. Cranio. 2018; 36(4): 243-249.

30. Braido GVV, Campi LB, Jordani PC, Fernandes G, Gonçalves DAG. Temporomandibular Disorder, Body Pain and Systematic Diseases: Assessing Their Associations in Adolescents. J Appl. Oral Sci. 2020; 28: 1-10.

31. Ouanounou A, Goldberg M, Haas DA. Pharmacotherapy in Temporomandibular Disorders: A Review. J Can Dent Assoc. 2017; 83 (7): 1-8 